MAGNETIC EXCITATIONS IN ANTIFERROMAGNETICALLY COUPLED SUPERLATTICES: $\mathrm{Fe} / \mathrm{Mo}$ AND Fe/Cr*

M. Grimsdtich, S. Kumar, and Eric. E. Fullerton

Materials Science Division

Argonne National Laboratory, Argonne, IL 60439

The submitled manuacript that been authored by a
contractor of the U.S. Government under concract
No. W-31-109-ENG-38. Accordingly, the U.S.
Government retains a nonexclusive, royaly-free
lioence to publith or reproduce the pubtished form of
this contribution, $\alpha$ allow others to do oo, for U.S.
Government purposes.

ANL/MSD/CP- -78798

DE93 007894

Proceedings of the 7th Latin American Symposium on Surface Science, Bariloche, Argentina, November 15-20, 1992 (to be published in J. Phys. C: Condensed Matter)

\section{DISCLAIMER}

This report was prepared as an account of work sponsored by an agency of the United States Government. Neither the United States Government nor any agency thereof, nor any of their employees, makes any warranty, express or implied, or assumes any legal liability or responsibility for the accuracy, completeness, or usefulness of any information, apparatus, product, or process disclosed, or represents that its use would not infringe privately owned rights. Reference herein to any specific commercial product, process, or service by trade name, trademark, manufacturer, or otherwise does not necessarily constitute or imply its endorsement, recommenciation, or favoring by the United States Government or any agency thereof. The views and opinions of authors expressed herein do not necessarily state or reflect those of the United States Government or any agency thereof.
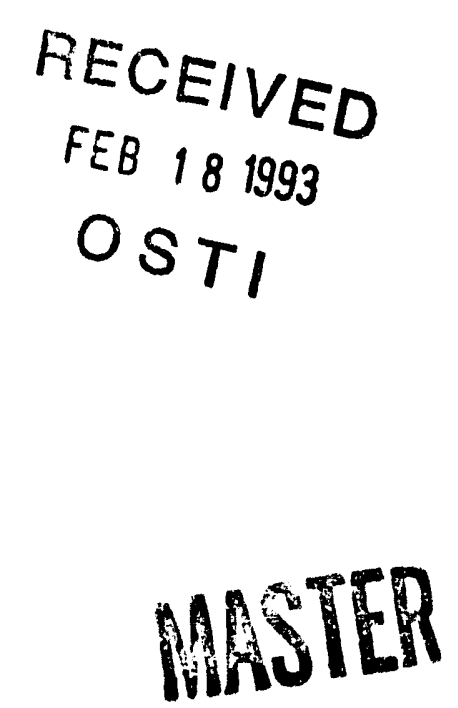

\footnotetext{
*Work supported by the U.S. Department of Energy, BES-Materials Sciences under contract \#W-31-109-ENG-38.
} 


\title{
Magnetic Excitations in Antiferromagnetically Coupled Superlattices: Fe/Mo and Fe/Cr.
}

\author{
M. Grimsditch, S. Kumar and Eric E. Fullerton \\ Materials Science Division, Argonne National Laboratory \\ Argonne IL 60439
}

Here we report the results of a Brillouin light scattering (BLS) study of magnetic excitations in antiferromagnetically (AF) coupled $\mathrm{Fe} / \mathrm{Mo}$ and $\mathrm{Fe} / \mathrm{Cr}$ superlattices. This technique has already been applied to $\mathrm{AF}$ coupled tri-layer films ${ }^{1-1}$ and shows that indeed these systems possess characteristics reflecting the coupling (both ferromagnetic and antiferromagnetic) between the ferromagnetic layers. However since in 'ordinary' magnetic superlattices it was found both theoretically5,6 and experimentally 7 that new collective modes exist resulting from dipolar coupling of the ferromagnetic layers, it could be expected that these new antiferromagnetic systems will also show novel behaviour. The experimental details relating to the sputtering growth conditions and BLS technique are discussed elsewhere.8,9 In this manuscript, only the magnetization and BLS results will be presented and discussed.

Figure 1 shows the in-plane magnetization loops measured using a squid magnetometer on two of our Fe/Mo samples; part a shows that the layers are AF coupled, part $\mathrm{b}$ indicates that the layers are either uncoupled or ferromagnetically coupled. The light arrows indicate the orientation of the magnetization in the Fe layers which is inferred from the data. The region of most obvious interest in Fig. $1 \mathrm{a}$ is that close to zero field where the layers are aligned antiferromagnetically. Fig. 2 shows BLS spectra recorded on the AF coupled sample at the fields indicated by the bold arrows in Fig. 1. The general trend is that in the region of most interest the spectra are of very poor quality and do not allow any useful information to be extracted. Only in the region of fields in which the layers are aligned ferromagnetically by the external field do the spectra show any clear features, usually two peaks on one side of zero and one on the other. 
The interpretation of the features in the spectra requires theoretical input which will be discussed later. However in the saturated regime it is reasonable to expect these systems to behave in a manner similar to an uncoupled magnetic superlattice. In this case the highest mode is a surface magnon and the lower frequency corresponds to the band of bulk modes. Since the surface mode represents a single excitation, data analysis should be more straightforward. Furthermore, the surface magnon frequency is expected to depend linearly on the applied field $(\mathrm{H})$. Figure 3 shows the magnetic field dependence of the two modes in a Fe/Mo sample. Within our experimental accuracy, the shift of the upper mode is linear with field while the lower mode shows some nonlinearity. The theoretically predicted slope for an uncoupled superlattice assuming the literature value for $\mathrm{Fe}$ is $2.98 \mathrm{GHz} / \mathrm{KG}$. The measured slopes as a function of non-magnetic layer thickness for a series of $\mathrm{Fe} / \mathrm{Mo}$ (dots) and $\mathrm{Fe} / \mathrm{Cr}$ (crosses) superlattices are shown in Fig. 4a. The solid line is the expected value for uncoupled layers. Shown in Fig. $4 \mathrm{~b}$ is the strength of the AF coupling deduced from the saturation field of the Fe/Mo Kerr magnetization loops. 10 Clearly the variation in the slope of the magnon frequency correlates well with the strength of the AF coupling.

Although a theory of magnons in saturated (i.e. the magnetization of the layers parallel to each other) superlattices with antiferromagnetic coupling exist, ${ }^{11}$ its complexity does not allow a simple interpretation of the results. It requires the search of the frequency for which the determinant of an $8 \mathrm{Nx} 8 \mathrm{~N}$ mairix is zero where $\mathrm{N}$ is the total number of magnetic layers. Apart from depending on the thicknesses of the layers and the wavevector of the magnons probed, the elements of the matrix depend on: applied field, two volume anisotropies, two surface anisotropies, bulk magnetization, exchange stiffness, strength of AF coupling and the g-factor. While some of these parameters can be 'fixed' from literature values or determined from separate single film measurements, various of the remaining parameters affect the slope of $\omega \mathrm{vs} \mathrm{H}$ and it is not possible to extract an unambiguous value of the AF coupling strength.

A number of attempts have been made to simplify the above theory: replacing the surface anisotropies by bulk anisotropies scaled by the inverse 
of the layer thicknesses, ${ }^{12}$ approximating the thickness of the non-magnetic layer by zero but retaining the variable coupling strength across it, 2 treating the magnetization of each layer as uniform, ${ }^{4}$ keeping only the exchange terms, 13 etc. Some of these approaches include the case in which the magnetization of the layers are not collinear. At this stage it appears that more, rigorous theoretical treatments of both the surface and bulk modes which are not as numerically intensive are needed. Furthermore quantitative comparisons of the AF coupling strengths determined by BLS and static magnetization measurements are required to test the validity of the assumptions.

In summary, although magnon spectra from AF coupled superlattices do show evidence of the indirect exchange coupling, it is not yet possible to extract any quantitative information from the experimental results.

Work supported by the US Department of Energy, BES-Materials Science, under contract \# W31-109-ENG-38.

\section{References}

1 P. Grünberg, R. Schreiber, Y. Pang, M. B. Brodsky and H. Sowers, Phys. Rev. Lett. 57, 2442 (1986)

2 J. Barnas and P. Grünberg, JMMM 82, 186 (1989)

3 M. Vohl, J. Barnas and P. Grünberg, Phys. Rev. B 39, 12003 (1989)

4 J. F. Cochran, J. Rudd,, W. B. Muir, B. Heinrich and Z. Celinski, Phys. Rev. B 42, 508 (1990)

$5 \quad$ P. Grünberg and K. Mika, Phys. Rev. B 27, 2955 (1983)

6 R. E. Camley, T. S. Rahman and D. L. Mills, Phys. Rev. B 27, 261 (1983)

7 M. Grimsditch, M. R. Khan, A. Kueny and I. K. Schuller, Phys. Rev. Lett. 51, 498 (1983)

8 J. E. Mattson, M. E. Brubaker, C. H. Sowers, M. Conover, Z. Qui and S. D. Bader, Phys. Rev. B 44, 9378 (1991)

9 J. R. Sandercock, in "Light Scattering in Solids III" ed by M. Cardona and G. Güntherodt (Springer-Verlag, Berlin, 1982)

10 M. E. Brubaker, J. E. Mattson, C. H. Sowers and S. D. Bader, Appl. Phys. Lett 58, 2306 (1991) 
11 B. Hillebrands, Phys. Rev. B 41, 530 (1990)

12 B. Hillebrands and G. Güntherodt, J. Appl. Phys. 61, 3756 (1987)

13 L. L. Hinchey and D. L. Mills, Phys. Rev. B 33, 3329 (1986) 


\section{Figure Captions}

Fig.1 Magnetization loops of two Fe/Mo superlattices showing antiferromagnetic and ferromagnetic coupling.

Fig.2 Brillouin spectra from the AF coupled $\mathrm{Fe} / \mathrm{Mo}(25 / 12)_{15}$ superlattice at fields where the magnetization of the layers are non-aligned and aligned respectively.

Fig. 3 Field dependence of the magnons in the $\mathrm{Fe} / \mathrm{Mo}(25 / 8)_{15}$ superlattice.

Fig. 4 a) Slope of the surface magnon peak as a function of the nonmagnetic layer thickness in $\mathrm{Fe} / \mathrm{Mo}$ (dots) and $\mathrm{Fe} / \mathrm{Cr}$ (crosses) superlattices. b) AF coupling strength obtained from the magnetization data of Ref. 10 for $\mathrm{Fe} / \mathrm{Mo}$ samples. 


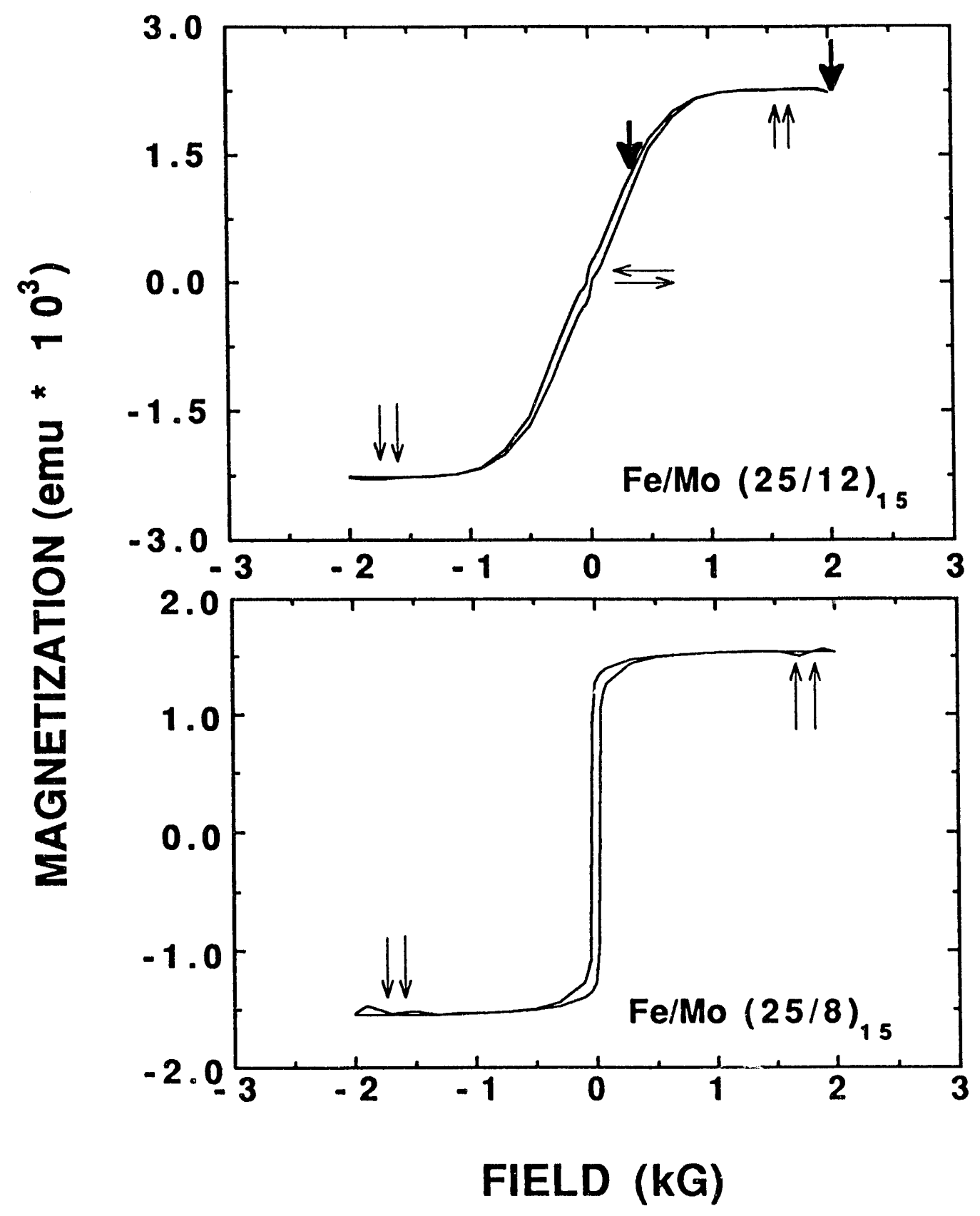




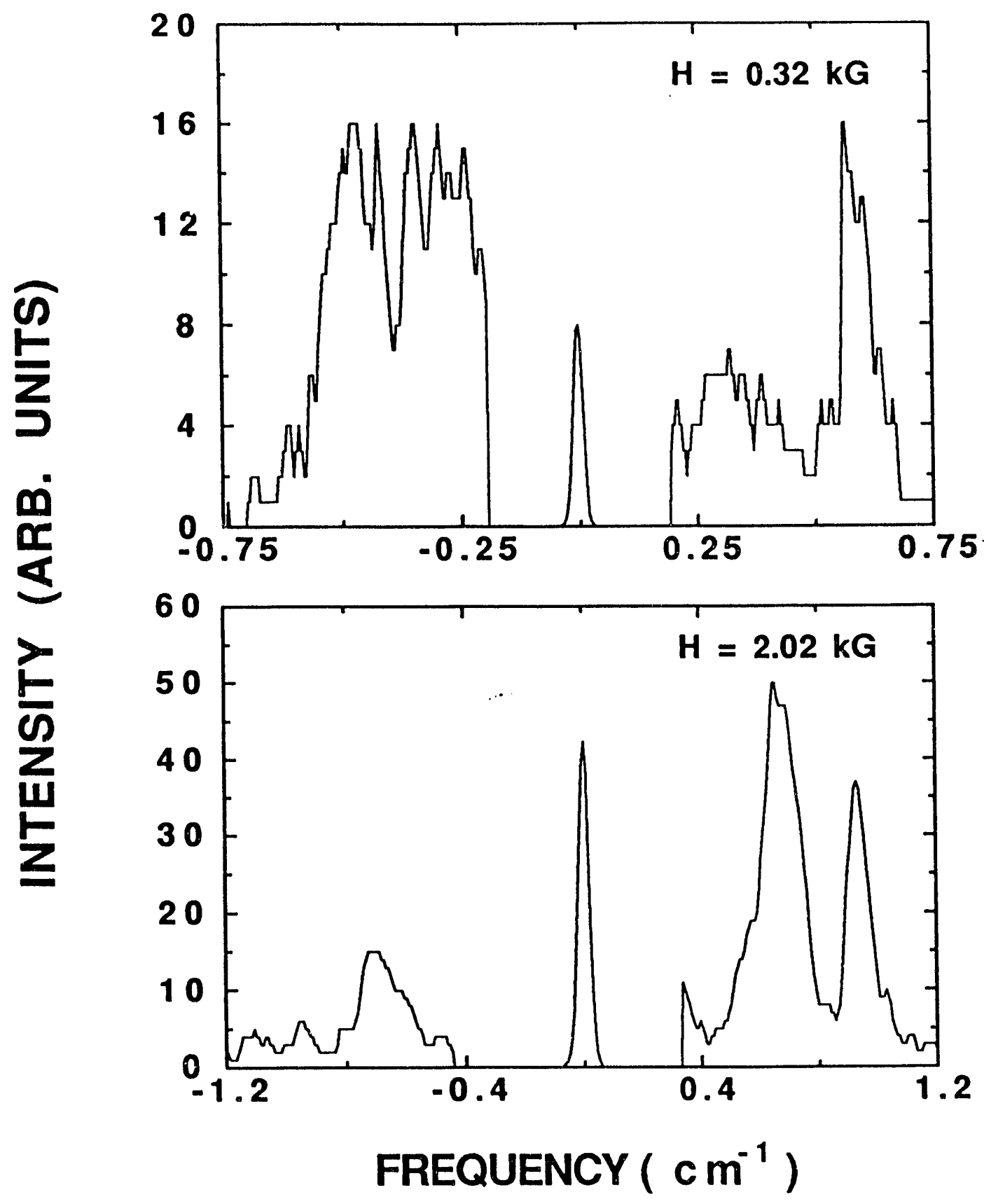




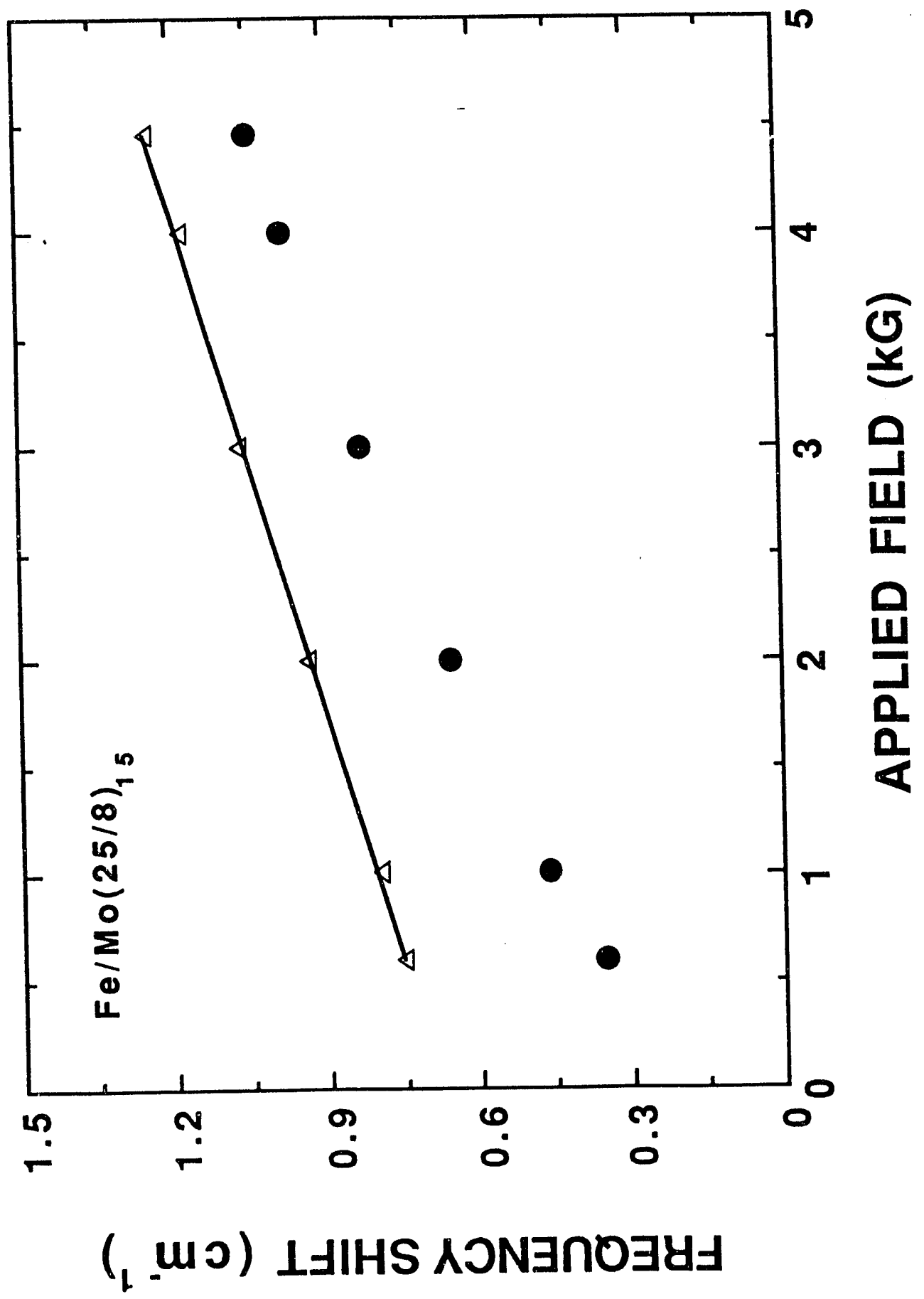



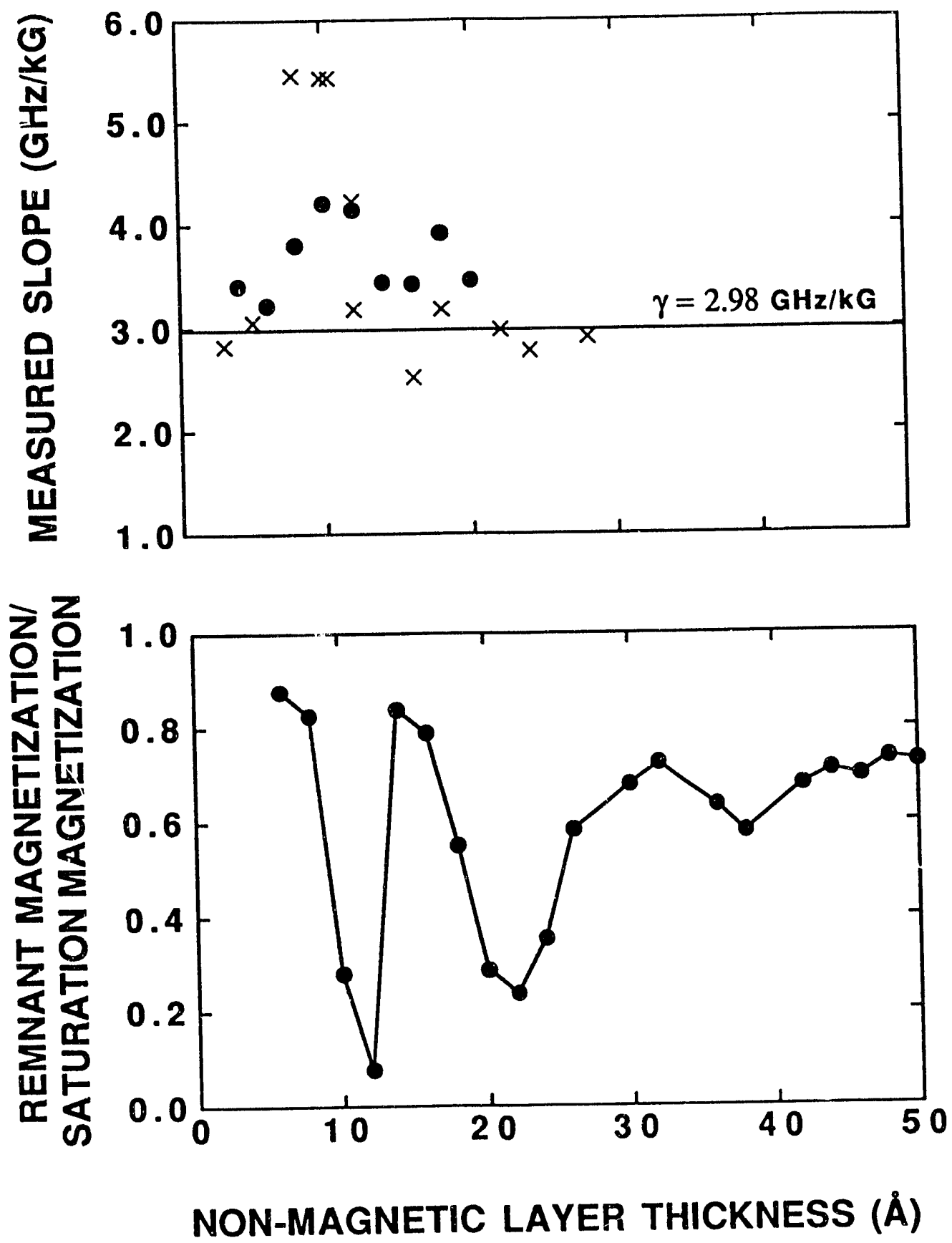

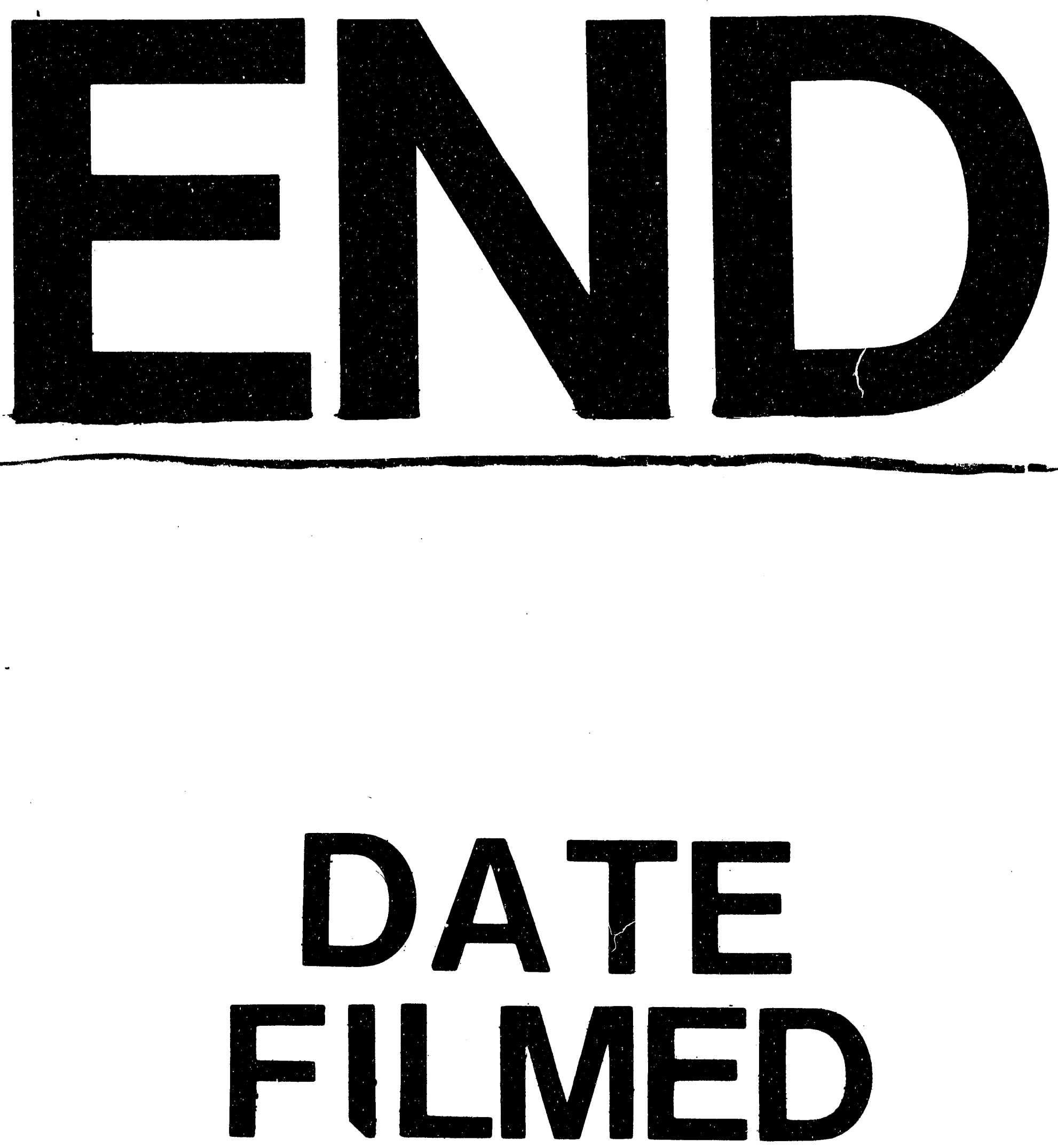

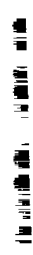

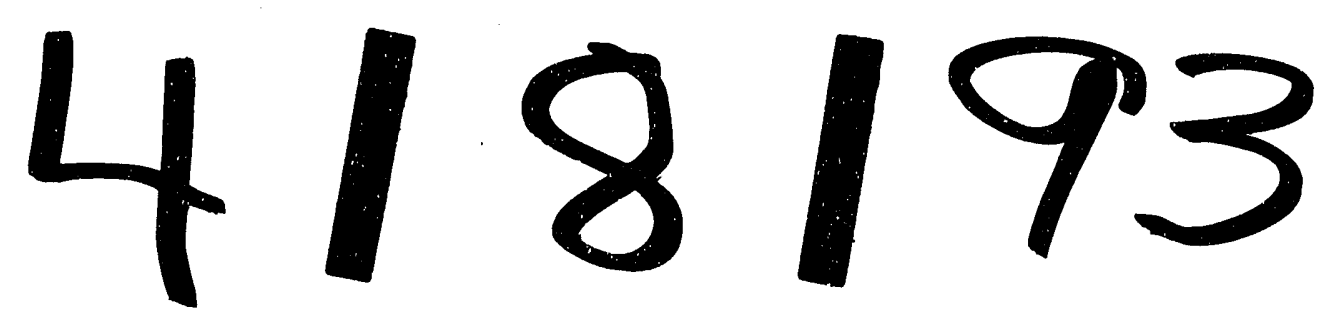




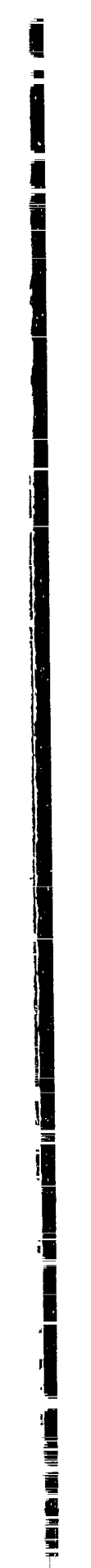

